\title{
Quercetin ameliorated insulin resistance via regulating METTL3-mediated N6-methyladenosine modification of PRKD2 mRNA in skeletal muscle and $\mathrm{C} 2 \mathrm{C} 12$ myocyte cell line
}

Jiao Yang

The Second Affiliated Hospital of Xi'an Jiaotong University

Albert Williams

The University of Hong Kong

Ning Wei ( $\nabla$ weining1988@live.cn )

Northwest Agriculture and Forestry University https://orcid.org/0000-0002-7790-7335

\section{Research}

Keywords: QUE, hyperinsulinemia, oxidative stress, glucose uptake, METTL3, methylation, GLUT4/IRS1/AKT pathway

Posted Date: February 28th, 2022

DOI: https://doi.org/10.21203/rs.3.rs-1364905/v1

License: (c) (1) This work is licensed under a Creative Commons Attribution 4.0 International License. Read Full License 


\section{Abstract}

Background: N6-Methyladenosine (m6A) is involved in many of pathological processes including insulin resistance (IR). Quercetin (Que), a bioactive compound with strong antioxidant activity, has potential therapeutic effects on IR-related metabolic diseases. The aim of this study is to investigate the roles of m6A and Que in hyperinsulinemia.

Methods: Male C57BI/ 6 mice received a high fat diet (HFD) for 8 weeks to establish an IR model. Biochemical analyzes, oral glucose tolerance test and homeostasis model assessment of IR (HOMA-IR) were conducted. C2C12 cells were transfected with methyltransferase-like 3 (METTL3) siRNA before treatment with palmitate (PA). 2-[N-(7-nitrobenz-2-oxa-1,3-diazol-4-yl) amino]-2-deoxyglucose (2-NBDG) was used to detect glucose uptake. Malondialdehyde (MDA) level, superoxide dismutase (SOD) activity, and glutathione (GSH) level were used to evaluate the levels of oxidative stress. SRAMP, a predicted site of m6A modification, was used to predict the relationship between METTL3 and erine-threonine kinase protein kinase D2 (PRKD2). Then, cells were treated with Que or/and transfected with pCDNA-METTL3 or PRKD2 siRNA. indirect calorimetry, Western blotting and qPCR were used to analyze protein and mRNA expression.

Results: Que treatment reduced the body weight, blood glucose, plasma TG and serum insulin, ameliorated IR, and decreased oxidative stress in HFD-fed mice. Que at noncytotoxic doses promoted PAinduced glucose uptake and inhibited oxidative stress in C2C12 cells. Moreover, METTL3 and PRKD2 was downregulated in PA-induced $\mathrm{C} 2 \mathrm{C} 12$ cells, and SRAMP revealed multiple m6A modification sites in the PRKD2 mRNA sequence. Downregulation of METTL3 enhanced PRKD2 expression by reducing m6A level and mRNA stability in PRKD2 mRNA transcript. C2C12 cells treatment with Que after inducing with PA inhibited m6A, METTL3 and phosphorylated insulin receptor substrate 1 ( $p$-IRS1) levels, increased the protein expression of PRKD2, glucose transporter type 4 (GLUT4) and p-AKT and glucose uptake, and reduced oxidative stress. However, METTL3 overexpression or PRKD2 silence reversed the inhibitory effects of Que on the levels of MDA, GLUT4 and p-AKT, and the promotive effects on glucose uptake, SOD, GSH and p-IRS1 levels.

Conclusion: Que promoted glucose uptake, repressed oxidative stress and improved IR through METTL3mediated N6-methylation of PRKD2 mRNA.

\section{Background}

Hyperinsulinemia is a compensatory response to insulin resistance (IR), which results from excessive levels of insulin in the blood [1,2]. Subjects with hyperinsulinism are often accompanied by a series of metabolic syndromes, including obesity, impaired glucose tolerance, hypertension and diabetes mellitus [3]. IR reduced glucose uptake, destroyed insulin signaling, disrupted glucose entry into the adipocytes, decreased glycogen synthesis and storage, and failed to suppress glucose production in response to 
insulin[4]. Hence, ameliorating IR is an effective way to treat hyperinsulinemia and a series of metabolic syndrome.

Oxidative stress is associated with a variety of metabolic diseases, including diabetes, hypertension and excess weight [5]. Study reported that hyperacetylation of the mitochondrial antioxidant superoxide dismutase 2 (SOD2) could lead to SOD2 inactivation and mitochondrial oxidative stress [6]. Oxidative stress mediated by high glucose was one of the major complications of diabetes mellitus[7]. Oxidative stress is also closely related to IR, because it can disrupt insulin signal transduction, cause the disorder of fat factors and induce IR[5]. Moreover, oxidative stress also can reduce insulin sensitivity[8]. Studies reported that oxidative stress may damage insulin secretion and insulin function in diabetic patients [9].

As the most prevalent internal chemical modification for mRNAs in eukaryotic cells, N6-methyladenosine (m6A) is catalyzed by a methyltransferase complex composed of methyltransferase like 3 (METTL3), methyltransferase like 14 (METTL14), and wilms tumor 1-associated protein (WTAP)[10]. m6A is involved in regulating many important biological processes, including cancer, cardiovascular and metabolic diseases[11]. However, the role of m6A in IR is still unclear.

Flavonoids are the most widespread polyphenols in human diet and have many biological activities. As one number of flavonoids, the mechanisms of quercetin (QUE) are pleiotropic[12]. QUE could increase the expression of endothelial nitric oxide synthase (eNOS), decrease the level of inducible nitric oxide synthase (iNOS), and thus alleviate insulin resistance in diabetic rats[13]. In addition, QUE also has been reported to have strong antioxidant and anti-inflammatory effects, and has multiple protective effects on oxidative stress-related diseases, including diabetes, cardiovascular diseases, atherosclerosis and cancer[14]. Satyakumar et al. reported that QUE effectively reversed the symptoms of fatty liver by decreasing triacyl glycerol accumulation, IR, inflammatory cytokine secretion and increasing cellular antioxidants in osteoarthritis induced hepatic steatosis of C2C12 cells[15], but the distinct molecular mechanisms of QUE in IR and oxidative stress is unclear. Hence, in this study, palmitate (PA)-induced IR C2C12 cell model and high-fat diet (HFD)-induced mouse model were established to investigate the effect and underlying mechanism of QUE on IR.

\section{Results}

\section{QUE ameliorated HFD-induced metabolic disorders and IR of mice}

Compared with control group, the body weight, fasting plasma glucose, and plasma TG of mice fed with HFD for 10 weeks were significantly increased (Fig. 1A-C, $P<0.05$ ). Furthermore, the degree of fasting insulin and HOMA-IR in HFD fed mice was signally higher than that in normal diet fed mice (Fig. 1D and $E, P<0.05)$. In accordance, HE staining showed that obviously lipid vacuolation in hepatic tissues from HFD mice when compared to the mice of control group (Fig. 1F). QUE treatment could lose weight to some extent, reduce blood glucose, plasma TG, serum insulin and HOMA-IR, and attenuate the HFD- 
induced hepatic steatosis (Fig. 1A-E, $P<0.05)$. After glucose or insulin injection, the HFD group had significantly higher blood glucose and area under the curve than the control group, whereas QUE treatment significantly alleviated mouse glucose tolerance and insulin resistance (Fig. 1G-J, P< 0.05).

\section{QUE ameliorated IR through inhibiting oxidative stress and METTL3-mediated N6-methyladenosine modification in skeletal muscle}

In relation to control group, mice fed HFD notably reduced SOD (Fig. $2 \mathrm{~A}, \mathrm{P}<0.05$ )

and GSH (Fig. 2B, $\mathrm{P}<0.05$ ) levels, increased ROS content (Fig. $2 \mathrm{C}, \mathrm{P}<0.05)$, elevated m6A methylated RNA level (Fig. 2D, $P<0.05$ ) and METTL3 mRNA and protein expression (Fig. 2E and F, $P<0.05$ ), inhibited GLUT4 and p-AKT protein expression, and increased p-IRS1 protein expression (Fig. $2 \mathrm{G}$ and $\mathrm{H}, P<0.05$ ). However, QUE treatment could markedly reverse the promotive effect on ROS content, m6A methylated RNA level, and METTL3 mRNA and protein levels and p-IRS1 protein expression, and the inhibitory effects of SOD and GSH levels, and protein expression of GLUT4 and p-AKT $(P<0.05)$.

\section{QUE ameliorated glucose uptake and suppressed oxidative stress in PA-induced C2C12 cells}

As shown in Fig. 3A, when the concentration of QUE was $20 \mu \mathrm{M}$, the viability of $\mathrm{C} 2 \mathrm{C} 12$ cells was significantly inhibited $(P<0.05)$, whereas the other concentrations had no effect. Therefore, we chose lower concentrations ( $5 \mu \mathrm{M}$ and $10 \mu \mathrm{M})$ of QUE for subsequent experiments. Figure 3B showed that cells treatment with $250 \mu \mathrm{M}$ PA could reduce 2-NBGD uptake, while $100 \mathrm{nM}$ insulin prominently increased the 2-NBGD uptake in C2C12 cells in relation to cells without insulin stimulation $(P<0.05)$. However, cells treatment with $250 \mu \mathrm{M}$ PA and $100 \mathrm{nM}$ insulin could not increase 2-NBGD uptake, which revealed that PA could stimulate IR $(P<0.05)$. Compared with $P A+$ insulin group, 2-NBGD uptake was increased after cells were treated with $5 \mu \mathrm{M}$ or $10 \mu \mathrm{M}$ QUE, and the increased effect of $10 \mu \mathrm{M}$ QUE was more obvious (Fig. 2B, $P<0.05)$. Cells induced by PA could significantly increase ROS content and METTL3 protein expression, and inhibit SOD activity and GSH level (Fig. 2C-G, P<0.05), while QUE treatment could reverse the increased effect of PA on ROS level and METTL3 protein level, and the inhibitory effect of SOD and GSH levels $(P<0.05)$. With the increase of QUE concentration, its inhibitory or promoting effect was more significant.

\section{Downregulation of METTL3 increased glucose uptake and suppressed oxidative stress in PA-induced $\mathrm{C} 2 \mathrm{C} 12$ cells}

C2C12 cells were transfected with METTL3 siRNA to investigate its role in glucose uptake and oxidative stress. The protein level of METTL3 was noticeably decreased after transfection with METTL3 siRNA in comparison with PA + Scramble group (Fig. $4 \mathrm{~A}$ and $\mathrm{B}, P<0.05$ ). Silencing METTL3 reversed the increase effect of PA on ROS content (Fig. 4F, P<0.05), and the inhibitory effect on glucose uptake (Fig. 4C, $\mathrm{P}<$ 0.05 ) and the levels of SOD and GSH (Fig. 4D and E, $P<0.05$ ). 


\section{Overexpression of METTL3 reversed the improvement effect of QUE on PA-induced glucose uptake and oxidative stress}

The protein expression of METTL3 was observably reduced after $\mathrm{C} 2 \mathrm{C} 12$ cells were treated with $10 \mu \mathrm{M}$ QUE compared with $250 \mu \mathrm{M}$ PA treatment (Fig. 5A and B, $P<0.05$ ). However, overexpression of METTL3 could reverse the inhibitory effect of $10 \mu \mathrm{M}$ QUE on the protein level of METTL3 $(P<0.05)$. Moreover, upregulation of METTL3 could also reverse the promotive effect of $10 \mu \mathrm{M}$ QUE on glucose uptake (Fig. 5C, P<0.05) and the levels of SOD (Fig. 5D, P<0.05) and GSH (Fig. 5E, P<0.05), and the inhibitory effect on ROS content (Fig. 5F, $\mathrm{P}<0.05$ ).

\section{METTL3 inhibited PRKD2 expression through its m6A demethylase activity}

To explore how QUE and METTL3 affect PA-induced glucose uptake and oxidative stress, m6A level and PRKD2 expression were detected in $\mathrm{C} 2 \mathrm{C} 12$ cells. The m6A level was increased after $\mathrm{C} 2 \mathrm{C} 12$ cells treated with $250 \mu \mathrm{M}$ PA (Fig. 6A, P< 0.05), while the mRNA, protein expression and fluorescence intensity of PRKD2 were decreased (Fig. 6B-D, $\mathrm{P}<0.05$ ). Cells treated with $10 \mu \mathrm{M}$ QUE reduced m6A level and increased the mRNA, protein expression and fluorescence intensity of PRKD2, while overexpression of METTL3 could reverse all the inhibitory effects of QUE $(P<0.05)$. SRAMP, a predicted site of m6A modification, revealed multiple m6A modification sites in the PRKD2 mRNA sequence (Fig. 6E). m6A MeRIP-qPCR revealed that METTL3 silence significantly increased PRKD2 mRNA enrichment in the antim6A group compared with the IgG group (Fig. 6F, $P<0.05$ ). Moreover, downregulation of METTL3 markedly increased the protein level and fluorescence intensity of PRKD2 (Fig. $6 \mathrm{G}$ and $\mathrm{H}, P<0.05$ ).

\section{Silence of PRKD2 reversed the improvement effect of QUE on PA-induced glucose uptake and oxidative stress through GLUT4/IRS-1/AKT pathway}

C2C12 cells were transfected with PRKD2 siRNA to analyze the role of PRKD2 in glucose uptake and oxidative stress induced by PA. The protein level of PRKD2 was dramatically decreased after transfection with PRKD2 siRNA by comparison with QUE treatment (Fig. 7A and $B, P<0.05$ ). Downregulation of PRKD2 counteracted the promotive effect of $10 \mu \mathrm{M}$ QUE on glucose uptake (Fig. $7 C, P<0.05$ ) and the levels of SOD and GSH (Fig. 7D and E, $P<0.05$ ), and the inhibitory effect on SOD content (Fig. 7F, $P<$ 0.05). Compared with control group, Western blot analysis revealed that the protein levels of GLUT4 and p-AKT were significantly reduced after PA stimulation, while the protein level of $p$-IRS1 was markedly increased (Fig. 7G and H, $P<0.05$ ). The levels of GLUT4 and p-AKT in PA and $10 \mu \mathrm{M}$ QUE group were increased, and p-IRS1 protein expression was reduced $(P<0.05)$. However, overexpression of METTL3 or 
silence of PRKD2 could reverse the promotive effect of QUE on the protein expression of GLUT4 and pAKT, and the inhibitory effect of p-IRS1 protein level $(P<0.05)$.

\section{Overexpression of METTL3 or silence of PRKD2 reversed the improving effects of QUE on HFD-induced metabolic disorders and IR}

By comparison with HFD + QUE group, overexpression of METTL3 or silence of PRKD2 could remarkedly increase mouse blood glucose (Fig. 8A, P<0.05), plasma TG (Fig. 8B, P<0.05), serum insulin (Fig. 8C, $\mathrm{P}<$ 0.05) and HOMA-IR (Fig. 8D, P<0.05). For GTT and IRT, METTL3 overexpression or PRKD2 silence dramatically increased blood glucose and area under the curve (Fig. 8E-H, $\mathrm{P}<0.05$ ). Moreover, overexpression of METTL3 or silence of PRKD2 increased the protein level of p-IRS1 and ROS content, and inhibited the protein expression of PRKD2, GLUT4 and p-AKT, and the levels of SOD and GSH $(P<$ 0.05).

\section{Discussion}

Recent studies showed that m6A methylation modification had a wide range of functional effects on homeostasis[16]. Our study revealed that HFD mice had significantly higher levels of blood glucose, TG and HOMA-IR, and the homeostasis of HFD mice were disrupted. Moreover, m6A levels were significantly increased in skeletal muscle of HFD mice. Abnormalities in any m6A level can lead to dysfunction and disease[17].

METTL3 is one of the core subunits of the methyltransferase complex catalyzing m6A formation, and aberrant expression of METTL3 is involved in many pathological processes, including IR[18]. Both m6A methylated RNA and METTL3 levels were consistently upregulated in liver tissues of Type 2 diabetes patients, and hepatocyte specific knockout of METTL3 improved insulin sensitivity and decreased fatty acid synthesis in HFD-fed mice[19]. Li et al reported that both METTL3 expression and m6A levels were increased in the liver of HFD-induced metabolic disorder mice. Overexpression of METTL3 aggravated HFD induced hepatic metabolic disorders and IR[20]. In our study, METTL3 and m6A levels were also upregulated in HFD-fed mice and PA-induced C2C12 cells, and silence of METTL3 could noticeably ameliorate IR and oxidative stress.

Flavonoids have been reported to promote insulin secretion. Rutin, the glycoside compound of QUE, increases insulin secretion in rat $\beta$ cells and maintain glucose sensing ability under high glucose conditions; it can also stimulate glucose transport into muscle by activating the synthesis and transport of transporter GLUT4[21]. QUE could acutely stimulate insulin release in rat and suppress INS-1 $\beta$ cell viability [22]. It also reports that QUE could inhibit intestinal glucose absorption, insulin secretion, and insulin sensitizing activity, as well as improve glucose utilization in peripheral tissues. Previous study suggested that QUE had poor bioavailability, while recent reports revealed that QUE can be detected in 
plasma after consumption of foods or supplements containing QUE components, indicating that QUE has a relatively long half-life in humans[12]. In addition, flavonoids and flavonols such as apigenin, luteolin, kaempferol, QUE, and fisetin can inhibit glucose transport, glycogen synthesis, protein synthesis, and cell survival by inhibiting IR phosphorylation through their activity at the C3 position[23].

QUE, a polyphenol with two aromatic rings, is the optimal configuration for free radical scavenging and transition metal ion binding, so QUE is considered to be a powerful antioxidant[24]. In nonalcoholic fatty liver, QUE ameliorated inflammation, oxidative stress and lipid metabolism in Db/Db mice [25]. QUE inhibited oxidative stress and inflammation progression by attenuating NF-KB and stabilizing HIF-1a, abrogating the possibility of hypobaric hypoxia induced pulmonary edema in rats[26]. Moreover, QUE ameliorated the body's antioxidant capacity by increasing the level of GSH and SOD, which utilized GSH as a hydrogen donor to convert free radicals generated in the body captured by SOD into $\mathrm{H}_{2} \mathrm{O}_{2}$ [27]. Hence, when $\mathrm{C} 2 \mathrm{C} 12$ cells were treated with QUE, C2C12 cell glucose uptake, SOD activity and the level of GSH increased, but ROS content decreased.

The serine-threonine kinase protein kinase D2 (PRKD2) deficiency causes to IR and metabolic disorders by promoting $\beta$-cell insulin secretion, which leads to hyperinsulinemia[28]. Our study revealed that PRKD2 level was downregulated in $\mathrm{C} 2 \mathrm{C} 12$ cells treated with $\mathrm{PA}$, while increased after transfected with pcDNAMETTL3. What is more, there are multiple m6A modification sites in the PRKD2 mRNA sequence through SRAMP. Hence, silencing PRKD2 could reverse the promotion of QUE on blood glucose absorption and the inhibition of oxidative stress. The reduction of GLUT4 expression or activity leads to reduced glucose uptake by skeletal muscle and adipocytes and is also an important molecular basis of IR[29]. Hyperbaric oxygen improves insulin sensitivity by promoting GLUT4 expression in muscle[30]. It is reported that any factor that reduces IRS-1 phosphorylation or induces serine IRS-1 phosphorylation at the 307 sites could damage insulin signal transduction and lead to insulin resistance[31]. Benzyl isothiocyanate ameliorated IR via inhibiting phosphorylation of IRS-1, reducing oxidative damage and upregulating the expression of GLUT4[32]. In our study, QUE could increase GLUT4 and p-AKT protein levels, and reduce p-IRS-1 protein expression. Overexpression of METTL3 or silence of PRKD2 reversed the promotive effects of QUE on GLUT4 and p-AKT protein expression and the inhibitory effect on p-IRS1 protein level.

\section{Conclusions}

Our study revealed that noncytotoxic doses of QUE could ameliorate IR and repress oxidative stress in skeletal muscle of HFD-fed mice and PA-stimulated insulin-resistant C2C12 cells through METTL3mediated N6-methylation of PRKD2 mRNA, suggesting that QUE may be a potential therapeutic target against hyperinsulinemia.

\section{Materials And Methods}

\section{Animals}


Male C57BL/ 6 mice at 3 months of age were obtain from laboratory animal center of Medicine College of $X i^{\prime} a n$ Jiaotong University and kept in the sterile cages under controlled conditions $\left(20 \pm 2^{\circ} \mathrm{C}\right.$, humidity $40-$ $50 \%$, cycle $12 \mathrm{~h} / 12 \mathrm{~h}$ light/dark) with free access to food and water. Mice were randomly divided into six groups ( $n=8$ per group): control group, high fat diet (HFD) group, HFD + QUE group, HFD + QUE + Lenti-NC + Scramble group, HFD + QUE + Lenti-METTL3 + Scramble group and HFD + QUE + Lenti-NC + PRKD2 siRNA group. The mice of control group were feed with a normal chow diet, which contained $70 \%$ carbohydrate calories, $20 \%$ protein calories, and $10 \%$ fat calories. HFD contained $20 \%$ carbohydrate calories, $20 \%$ protein calories, and $60 \%$ fat calories. For QUE treatment, HFD-fed mice for 8 weeks were intraperitoneally injected with QUE (10 mg/kg) daily for 10 weeks. Lenti-METTL3 and Lenti-NC were purchased from GeneChem (Shanghai, China) and injected to mice via the tail vein once a week for four weeks $\left(1 \times 10^{9}\right.$ units $\left./ 100 \mu \mathrm{L}\right)$. Small interfering RNA targeting serine-threonine kinase protein kinase D2 (PRKD2 siRNA) were injected intraperitoneally into mice at $2 \mathrm{mg} / \mathrm{Kg}$. All mice were fasted for $12 \mathrm{~h}$ before sampling. After experiments, the mice were anaesthetized with an intraperitoneal injection of pentobarbital sodium ( $80 \mathrm{mg} / \mathrm{Kg}$ ), and were euthanized with an intraperitoneal injection of excessive sodium pentobarbital. The research was approved by the ethics committee of the Second Affiliated Hospital of Xi'an Jiaotong University.

\section{Cell Lines and reagents}

Mouse myoblast cell line $\mathrm{C} 2 \mathrm{C} 12$ was purchased from Shanghai Institute of Biochemistry and Cell Biology (Shanghai, China). Cells were cultured in Dulbecco's Modified Eagles Medium (DMEM, Gibco, Rockville, MD), containing with $10 \%$ fetal bovine serum (FBS, HyClone, Salt Lake City, UT) and $1 \%$ penicillinstreptomycin (Sigma, St. Louis, MO, USA) at $37^{\circ} \mathrm{C}$ in a humidified atmosphere of $5 \% \mathrm{CO}_{2}$. QUE (purity $\geq$ 95\%, CAS NO:117-39-5) and PA (purity $\geq 99.0 \%$, CAS NO: 57-10-3) were purchased from Sigma (St Louis, MO, USA). 2-[N-(7-nitrobenz-2-oxa-1,3-diazol-4-yl) amino]-2-deoxyglucose (2-NBDG) was provided by YUBO biologycal (Carlsbad, CA, USA). Human insulin was purchased from Santa Cruz Biotechnology (Shanghai, China).

\section{Glucose tolerance test (GTT) and insulin resistance test (IRT)}

Glucose and insulin tolerance tests were conducted in mice after fasting for one night. Briefly, mice were intraperitoneally injected with glucose at a dose of $2 \mathrm{~g} / \mathrm{Kg}$ body weight, and blood samples were collected from the tail tip at the time points of $30,60,90$ and $120 \mathrm{~min}$. For IRT, the mice were received an intraperitoneal injection of $0.75 \mathrm{IU} / \mathrm{kg}$ insulin, and blood glucose concentrations were measured after 30 , 60, 90 and 120 min respectively. Glucose levels were analyzed with glucometer (Yuyan Instruments, Shanghai, China). The insulin resistance index was calculated using the equation: homeostasis model assessment of IR (HOMA-IR) = blood glucose $(\mathrm{mmol} / \mathrm{L}) \times$ serum insulin $(\mu \mathrm{U} / \mathrm{mL}) / 22.5$.

\section{Measurement of triglyceride (TG), superoxide dismutase (SOD), antioxidant no-enzymatic (GSH) and oxygen species (ROS)}


TG levels from mouse plasma were measured using an ELISA kit (ab65336, Abcam, Cambridge, UK). The levels of SOD and GSH from $\mathrm{C} 2 \mathrm{C} 12$ cell suspensions and mouse skeletal muscle lysates were measured using ELISA under standard conditions, according to the manufacturer's instructions on the reagent kits. ROS content was detected with ROS assay kit (ab113851, Abcam, Cambridge, UK). The absorbance at the corresponding wavelength was measured with a microplate reader (Molecular Devices, Sunnyvale, CA, USA).

\section{Cell viability assay}

Cell survival experiment was performed with a cell counting kit-8 (CCK-8) assay (Bio-Rad, Hercules, CA, USA). Cells were treated with different concentrations of QUE (final concentrations of $0,5,10,15,20 \mu \mathrm{M}$ ). After $24 \mathrm{~h}$ of co-incubation, $10 \mu \mathrm{L}$ of CCK-8 solution was added to each well for another $4 \mathrm{~h}$ at $37^{\circ} \mathrm{C}$. The absorption values were detected with a microplate reader (Molecular Devices, Sunnyvale, CA, USA) at 450 $\mathrm{nm}$.

\section{Cell transfection}

Overexpression plasmid of METTL3 (pcDNA-METTL3), small METTL3 siRNA, PRKD2 siRNA, and their corresponding negative controls were purchased from RiboBio (Guangzhou, China). The siRNA sequences were as follows: METTL3 siRNA, 5'-GGC ACU UGG AUU UAA GGA ATT-3'; PRKD2 siRNA, 5'AAG UCU CAG GAG CGA UGG CAC-3'; Scramble, 5'-CGA GGG ATG AGC CCG CGT AG-3'. Cell transfection were performed with the Lipofectamine 3000 Transfection Reagent (Invitrogen, Carlsbad, USA) according to the manufacturer's instructions. After transfection for $48 \mathrm{~h}$, cells were harvested for subsequent experiments.

\section{Glucose uptake assay}

Glucose uptake assay was performed using the fluorescent D-glucose analog 2-NBDG to monitor intracellular glucose uptake. C2C12 cells were seeded in 96 -well plates at a density of $4 \times 10^{5}$ cells/well for $24 \mathrm{~h}$. Then cells were incubated with 2-NDBG (a final concentration of $100 \mathrm{mM}$ ) for another $2 \mathrm{~h}$. After different treatments, free 2-NBDG was washed with PBS and then $200 \mu \mathrm{L}$ of DMSO was added to each well to lyse the cell membrane. The fluorescence intensities were measured at an excitation wavelength of $488 \mathrm{~nm}$ and an emission wavelength of $520 \mathrm{~nm}$ with a fluorescence microplate reader (Molecular Devices, CA, USA).

\section{Reverse Transcription-quantitative PCR (RT-qPCR)}

Total RNA from skeletal muscle tissues or cells was extracted using Trizol reagent (Invitrogen, Carlsbad, USA) under the manufacturer's instructions. TaqMan microRNA Reverse Transcription Kit (Applied Biosystems, Foster City, USA) was used to synthesize cDNA in a $25 \mu \mathrm{L}$ reaction volume containing 12.5 $\mu \mathrm{L}$ SYBR Premix Ex Taq $\otimes, 1.0 \mu \mathrm{L}$ of RT primer, and $1 \mu \mathrm{L}$ cDNA sample, and $10.5 \mu \mathrm{L}$ double distilled $\mathrm{H}_{2} \mathrm{O}$. RT-qPCR was performed at $95^{\circ} \mathrm{C}$ for $2 \mathrm{~min}$, followed by 40 circles at $95^{\circ} \mathrm{C}$ for $15 \mathrm{~s}$ and $30 \mathrm{~s}$ at $60^{\circ} \mathrm{C}$ and dissociation at $95^{\circ} \mathrm{C}$ for $30 \mathrm{~s}$. GAPDH was acted as an internal reference. Data were analyzed using the $2^{-\triangle \triangle C T}$ method. The following primers were used: METTL3 (forward 5'-3': AGC CTT CTG AAC CAA CAG 
TCC; reverse5'-3': CCG ACC TCG AGA GCG AAAT); PRKD2 (forward 5'-3': AGA GCC AGG TAA CAG GAA CAA TAG; reverse 5'-3': GTG CTA AGG AGG GAG GCT CT); GAPDH (forward 5'-3': GGA CCT GAC CTG CCG TCT AG; reverse 5'-3': GTA GCC CAG GTA GCC CTT GA).

\section{m6A MeRIP-qPCR}

Total RNA from C2C12 cells was isolated using PolyATtract mRNA isolation systems (Promega, Madison, USA). Then, mRNA was denatured at $70^{\circ} \mathrm{C}$ for $10 \mathrm{~min}$, and $5 \mu \mathrm{L}$ of anti-m6A antibody (ab208577, Abcam, Cambridge, UK) or anti-lgG (ab172730, Abcam, Cambridge, UK) was conjugated to protein A/G magnetic beads in IP buffer ( $150 \mathrm{mM} \mathrm{NaCl}, 10 \mathrm{mM}$ Tris-HCL, and $0.1 \% \mathrm{NP}-40)$ for $2 \mathrm{~h}$ at $4{ }^{\circ} \mathrm{C}$. A total of $100 \mathrm{ng} \mathrm{RNA}$ was incubated with antibodies in a buffer containing RNase inhibitor and protease inhibitor. m6A RNA was eluted with $6.7 \mathrm{mM}$ of N6-methyladenosine 5'-monophosphate sodium salt (Sigma, St. Louis, MO, USA) for $1 \mathrm{~h}$ at $4{ }^{\circ} \mathrm{C}$. Total RNA was eluted with elution buffer and purified by phenol chloroform extraction. The enrichment of m6A was determined with RT-qPCR analysis. Fold enrichment was calculated by calculating the $2^{-\Delta C t}$ of eluate relative to the input sample.

\section{Total RNA methylation quantification}

Total RNA from C2C12 cells was extracted using TRIzol reagent (Invitrogen, Carlsbad, USA). m6A level in the total RNA was measured with a commercial m6A RNA methylation quantification kit (ab185912; Abcam, Cambridge, UK). In briefly, 200 ng of RNAs were added into each assay well, followed by a capture antibody solution and a detection antibody solution, respectively. Colorimetric quantification was analyzed through reading the absorbance at $450 \mathrm{~nm}$ wavelength, and the level of $\mathrm{m} 6 \mathrm{~A}$ of each sample was colourimetrically measured according to the standard curve.

\section{Hematoxylin-Eosin (HE) Staining}

Liver tissues were fixed in $4 \%$ paraformaldehyde overnight and then embedded in paraffin. Slices of liver sections were dewaxed with xylene, then dehydrated in ethanol. The slices were stained with HE (Synthgene, Nanjing, China). The histological images were captured by an inverse microscope equipped with an LCD camera (Olympus, Tokyo, Japan).

\section{Immunofluorescence analysis}

C2C12 cells were fixed with $4 \%$ paraformaldehyde for $15 \mathrm{~min}$ and then cells were permeabilized using Triton X-100 (0.1\%) and subsequently blocked with $5 \%$ goat serum for $2 \mathrm{~h}$ at room temperature. Next, primary antibody anti-PRKD2 (ab51250, Abcam, Cambridge, UK) was added and cultivated overnight at 4 ${ }^{\circ} \mathrm{C}$. Alexa fluor 488 fluorescein conjugated secondary antibody (ab150077, Abcam, Cambridge, UK) was replenished and incubated at room temperature for $1 \mathrm{~h}$. Finally, DAPI was incubated at room temperature for 10 min. Fluorescence intensity was evaluated by fluorescence microscopy (MF43-N, Mshot, China).

\section{Western blotting}

Total protein from skeletal muscle tissues and $\mathrm{C} 2 \mathrm{C} 12$ cells were extracted with Radio Immunoprecipitation Assay lysis buffer (Beyotime, Shanghai, China) for 30 min $4^{\circ} \mathrm{C}$, and protein 
concentration was quantified by using an Enhanced BCA Kit (Beyotime, Shanghai, China). Protein samples were separated on $10 \%$ sodium dodecyl sulfate polyacrylamide gel electrophoresis and transferred to polyvinylidene difluoride membranes (Millipore, Bedford, USA). After blocked with 5\% skim milk at room temperature for $1 \mathrm{~h}$, membranes were incubated with primary antibodies against METTL3 (1:1000, ab195352, Abcam, Cambridge, UK), PRKD2 (1:500, ab51250, Abcam, Cambridge, UK), GLUT4 (1:2000, ab33780, Abcam, Cambridge, UK), IRS1 (1:1000, ab232463, Abcam, Cambridge, UK), p-ser307IRS1 (1:250, ab1194, Abcam, Cambridge, UK), protein kinase B (AKT; 1:1000, ab8805, Abcam, Cambridge, UK), p-AKT (p-AKT; 1:1000, ab38449, Abcam, Cambridge, UK). Then membranes were washed by using TBST for 3 times and incubated with a horseradish peroxidase-conjugated secondary antibody (1:5000, ab6721, Abcam, Cambridge, USA) for $1 \mathrm{~h}$ at room temperature. After washing, chemiluminescent reagent was used to detect protein bands. And signals were visualized using a chemiluminescence imaging system (Bioshine ChemiQ 4800 mini, Oxiang, Shanghai, China).

\section{Statistical Analysis}

All experiment were repeated 3 times independently and all data were reported as the mean \pm SEM using GraphPad Prism 7.0 statistical software (GraphPad Software, La Jolla, CA, USA). One-way analysis of variance (ANOVA), followed by Tukey's multiple comparisons test was used for comparisons between three groups. ${ }^{\star \star} P<0.05$ was regarded significant difference.

\section{Abbreviations}

$\mathrm{m} 6 \mathrm{~A}$

N6-Methyladenosine

$\mathrm{IR}$

insulin resistance

QUE

Quercetin

HFD

high fat diet

PA

palmitate

METTL3

methyltransferase-like 3

METTL14

methyltransferase like 14

WTAP

wilms tumor 1-associated protein

PRKD2

protein kinase $\mathrm{D} 2$

p-IRS1 
phosphorylated insulin receptor substrate 1

GLUT4

glucose transporter type 4

p-AKT

phosphorylated protein kinase $B$

SOD2

superoxide dismutase 2

eNOS

endothelial nitric oxide synthase

iNOS

inducible nitric oxide synthase.

\section{Declarations}

\section{Acknowledgments}

Not applicable.

\section{Authors' contributions}

Jiao Y performed the experiments, statistical analysis and prepared the manuscript; Albert Williams analyzed and proofread the data and prepared the figures; Wei $\mathrm{N}$ participated in the study design and conceptualization, supervised and directed the work, drafted and revised the manuscript.

\section{Funding}

This study was supported by Key R\&D projects of Shaanxi provincial (2020SF-165).

\section{Availability of data and materials}

The datasets used or analyzed during the current study are available from the corresponding author on reasonable request.

\section{Ethics approval and consent to participate}

All subjects had not received preoperative radiotherapy and/or chemotherapy and were obtained with informed consent before surgery. The protocols were approved by the Ethics Committee of the Second Affiliated Hospital of Xi'an Jiaotong University.

\section{Consent for publication}

The manuscript is approved by all authors for publication.

\section{Competing interests}


The authors declare that they have no competing interests.

\section{References}

1. Adeghate E, Singh J: Structural changes in the myocardium during diabetes-induced cardiomyopathy. Heart failure reviews 2014, 19(1):15-23.

2. Jia G, DeMarco VG, Sowers JR: Insulin resistance and hyperinsulinaemia in diabetic cardiomyopathy. Nat Rev Endocrinol 2016, 12(3):144-153.

3. Yaribeygi H, Farrokhi FR, Butler AE, Sahebkar A: Insulin resistance: Review of the underlying molecular mechanisms. J Cell Physiol 2019, 234(6):8152-8161.

4. Malik SA, Acharya JD, Mehendale NK, Kamat SS, Ghaskadbi SS: Pterostilbene reverses palmitic acid mediated insulin resistance in HepG2 cells by reducing oxidative stress and triglyceride accumulation. Free Radic Res 2019, 53(7):815-827.

5. Houstis N, Rosen ED, Lander ES: Reactive oxygen species have a causal role in multiple forms of insulin resistance. Nature 2006, 440(7086):944-948.

6. Dikalova AE, Pandey A, Xiao L, Arslanbaeva L, Sidorova T, Lopez MG, Billings FTt, Verdin E, Auwerx J, Harrison DG et al: Mitochondrial Deacetylase Sirt3 Reduces Vascular Dysfunction and Hypertension While Sirt3 Depletion in Essential Hypertension Is Linked to Vascular Inflammation and Oxidative Stress. Circulation research 2020, 126(4):439-452.

7. Takanche JS, Kim JE, Han SH, Yi HK: Effect of gomisin A on osteoblast differentiation in high glucose-mediated oxidative stress. Phytomedicine : international journal of phytotherapy and phytopharmacology 2020, 66:153107.

8. Furukawa S, Fujita T, Shimabukuro M, Iwaki M, Yamada Y, Nakajima Y, Nakayama O, Makishima M, Matsuda M, Shimomura I: Increased oxidative stress in obesity and its impact on metabolic syndrome. J Clin Invest 2004, 114(12):1752-1761.

9. Ighodaro OM: Molecular pathways associated with oxidative stress in diabetes mellitus. Biomed Pharmacother 2018, 108:656-662.

10. Sun T, Wu R, Ming L: The role of m6A RNA methylation in cancer. Biomedicine \& pharmacotherapy = Biomedecine \& pharmacotherapie 2019, 112:108613.

11. Chen Z, Wu L, Zhou J, Lin X, Peng Y, Ge L, Chiang CM, Huang H, Wang H, He W: N6-methyladenosineinduced ERRy triggers chemoresistance of cancer cells through upregulation of $A B C B 1$ and metabolic reprogramming. Theranostics 2020, 10(8):3382-3396.

12. Eid HM, Haddad PS: The Antidiabetic Potential of Quercetin: Underlying Mechanisms. Current medicinal chemistry 2017, 24(4):355-364.

13. Babacanoglu C, Yildirim N, Sadi G, Pektas MB, Akar F: Resveratrol prevents high-fructose corn syrupinduced vascular insulin resistance and dysfunction in rats. Food Chem Toxicol 2013, 60:160-167.

14. D'Andrea G: Quercetin: A flavonol with multifaceted therapeutic applications? Fitoterapia 2015, 106:256-271. 
15. Vidyashankar S, Sandeep Varma R, Patki PS: Quercetin ameliorate insulin resistance and upregulates cellular antioxidants during oleic acid induced hepatic steatosis in HepG2 cells. Toxicol In Vitro 2013, 27(2):945-953.

16. Xiang Y, Laurent B, Hsu CH, Nachtergaele S, Lu Z, Sheng W, Xu C, Chen H, Ouyang J, Wang S et al: RNA m(6)A methylation regulates the ultraviolet-induced DNA damage response. Nature 2017, 543(7646):573-576.

17. Chen M, Wei L, Law CT, Tsang FH, Shen J, Cheng CL, Tsang LH, Ho DW, Chiu DK, Lee JM et al: RNA N6-methyladenosine methyltransferase-like 3 promotes liver cancer progression through YTHDF2dependent posttranscriptional silencing of SOCS2. Hepatology (Baltimore, Md) 2018, 67(6):22542270 .

18. Wu Z, Shi Y, Lu M, Song M, Yu Z, Wang J, Wang S, Ren J, Yang YG, Liu GH et al: METTL3 counteracts premature aging via m6A-dependent stabilization of MIS12 mRNA. Nucleic acids research 2020, 48(19):11083-11096.

19. Xie W, Ma LL, Xu YQ, Wang BH, Li SM: METTL3 inhibits hepatic insulin sensitivity via N6methyladenosine modification of Fasn mRNA and promoting fatty acid metabolism. Biochemical and biophysical research communications 2019, 518(1):120-126.

20. Li Y, Zhang Q, Cui G, Zhao F, Tian X, Sun BF, Yang Y, Li W: m(6)A Regulates Liver Metabolic Disorders and Hepatogenous Diabetes. Genomics, proteomics \& bioinformatics 2020.

21. Ghorbani A: Mechanisms of antidiabetic effects of flavonoid rutin. Biomedicine \& pharmacotherapy = Biomedecine \& pharmacotherapie 2017, 96:305-312.

22. Kittl M, Beyreis M, Tumurkhuu M, Fürst J, Helm K, Pitschmann A, Gaisberger M, Glasl S, Ritter M, Jakab M: Quercetin Stimulates Insulin Secretion and Reduces the Viability of Rat INS-1 Beta-Cells. Cellular physiology and biochemistry : international journal of experimental cellular physiology, biochemistry, and pharmacology 2016, 39(1):278-293.

23. Nomura M, Takahashi T, Nagata N, Tsutsumi K, Kobayashi S, Akiba T, Yokogawa K, Moritani S, Miyamoto K: Inhibitory mechanisms of flavonoids on insulin-stimulated glucose uptake in MC3T3G2/PA6 adipose cells. Biological \& pharmaceutical bulletin 2008, 31(7):1403-1409.

24. Zhao L, Wu J, Yang J, Wei J, Gao W, Guo C: Dietary quercetin supplementation increases serum antioxidant capacity and alters hepatic gene expression profile in rats. Exp Biol Med (Maywood) 2011, 236(6):701-706.

25. Yang $H$, Yang T, Heng C, Zhou Y, Jiang Z, Qian X, Du L, Mao S, Yin X, Lu Q: Quercetin improves nonalcoholic fatty liver by ameliorating inflammation, oxidative stress, and lipid metabolism in db/db mice. Phytother Res 2019, 33(12):3140-3152.

26. Tripathi A, Kumar B, Sagi SSK: Prophylactic efficacy of Quercetin in ameliorating the hypoxia induced vascular leakage in lungs of rats. PLoS One 2019, 14(6):e0219075.

27. Granado-Serrano AB, Martin MA, Bravo L, Goya L, Ramos S: Quercetin modulates Nrf2 and glutathione-related defenses in HepG2 cells: Involvement of p38. Chem Biol Interact 2012, 195(2):154-164. 
28. Xiao Y, Wang C, Chen JY, Lu F, Wang J, Hou N, Hu X, Zeng F, Ma D, Sun X et al: Deficiency of PRKD2 triggers hyperinsulinemia and metabolic disorders. Nat Commun 2018, 9(1):2015.

29. McNay EC, Pearson-Leary J: GluT4: A central player in hippocampal memory and brain insulin resistance. Experimental neurology 2020, 323:113076.

30. Liu Y, Zhang D, Yuan J, Song L, Zhang C, Lin Q, Li M, Sheng Z, Ma Z, Lv F et al: Hyperbaric Oxygen Ameliorates Insulin Sensitivity by Increasing GLUT4 Expression in Skeletal Muscle and Stimulating UCP1 in Brown Adipose Tissue in T2DM Mice. Frontiers in endocrinology 2020, 11:32.

31. Hirosumi J, Tuncman G, Chang L, Gorgun CZ, Uysal KT, Maeda K, Karin M, Hotamisligil GS: A central role for JNK in obesity and insulin resistance. Nature 2002, 420(6913):333-336.

32. Chuang WT, Yen CC, Huang CS, Chen HW, Lii CK: Benzyl Isothiocyanate Ameliorates High-Fat DietInduced Hyperglycemia by Enhancing Nrf2-Dependent Antioxidant Defense-Mediated IRS1/AKT/TBC1D1 Signaling and GLUT4 Expression in Skeletal Muscle. Journal of agricultural and food chemistry 2020, 68(51):15228-15238.

\section{Figures}

\section{Figure 1}

QUE ameliorated HFD-induced metabolic disorders and IR of mice. Body weight (A), fasting blood glucose (B), plasma TG (C), serum insulin (D) and HOMA-IR (E) in each group ( $n=8$ per group). ( $F$ ) HE-stain liver sections. Scale bars $=10 \mu \mathrm{m}$. Blood glucose levels $(G)$ and AUC $(H)$ of HFD mice in response to the treatment of QUE according to GTT. Blood glucose levels (I) and AUC (J) of HFD mice in response to the treatment of QUE according to IRT. $(\mathrm{N}=3)$ Data are expressed as the mean \pm SEM. ** $P<0.05$ compared with control or HFD groups.

\section{Figure 2}

\section{QUE ameliorated IR through inhibiting oxidative stress and METTL3-mediated N6-methyladenosine} modification in skeletal muscle. The levels of SOD (A), GSH (B) and ROS (C) in skeletal muscle were analyzed. The levels of m6A (D), METTL3 mRNA (EB) and MELLT3 protein (F) in skeletal muscle were detected. Western blotting was used to analyze the protein expression of GLUT4, IRS1, p-IRS1, AKT and pAKT in skeletal muscle. $(\mathrm{N}=3)$ Data are expressed as the mean $\pm \mathrm{SEM} . * * P<0.05$ compared with control or HFD groups.

Figure 3 
QUE ameliorated glucose uptake and suppressed oxidative stress in PA-induced C2C12 cells. (A) Cell viability was detected through CCK-8 assay after $\mathrm{C} 2 \mathrm{C} 12$ cells treatment with different concentrations of QUE for 24h. (B) Effects of $250 \mu \mathrm{M}$ PA, $100 \mathrm{nM}$ insulin and different concentrations of QUE on 2-NBDG uptake. Effects of QUE on the levels of SOD (C), GSH (D) and ROS (E) after C2C12 cells were stimulated by PA and QUE. The levels of METTL3 mRNA $(F)$ and protein $(G)$ were analyzed with qPCR and Western blotting. $(\mathrm{N}=3)$ Data are expressed as the mean \pm SEM. ${ }^{*} P<0.05$ compared with control group, PA group, insulin group, or PA+5 $\mu \mathrm{M}$ QUE group.

\section{Figure 4}

Downregulation of METTL3 increased glucose uptake and suppressed oxidative stress in PA-induced C2C12 cells. C2C12 cells were treated with $250 \mu \mathrm{M}$ PA or transfected with METTL3 siRNA for $24 \mathrm{~h}$ before treatment with $250 \mu \mathrm{M}$ PA. (A-B) The protein level of METTL3 was detected using Western blot assay. (C) 2-NBDG uptake of C2C12 cells. The levels of SOD (D), GSH (E), and ROS (F) were detected. $(N=3)$ Data are expressed as the mean \pm SEM. ${ }^{* *} P<0.05$ compared with control group or PA +Scramble group.

\section{Figure 5}

Overexpression of METTL3 reversed the improvement effect of QUE on PA-induced glucose uptake and oxidative stress. C2C12 cells were transfected with pcDNA-METTL3 before treatment with $10 \mu \mathrm{M}$ QUE. (AB) The protein expression of METTL3 in C2C12 cells were detected using qPCR and Western blot assays. (C) 2-NBDG uptake of C2C12 cells. The levels of SOD (D), GSH (E), and ROS (F) in each group. (N=3) Data are expressed as the mean \pm SEM. ${ }^{\star *} P<0.05$ compared with PA group or PA+10 $\mu \mathrm{M}$ QUE group.

\section{Figure 6}

METTL3 inhibited PRKD2 expression through its m6A demethylase activity. C2C12 cells were transfected with pcDNA-METTL3 for $24 \mathrm{~h}$ before treatment with $10 \mu \mathrm{M}$ QUE. The levels of m6A (A), PRKD2 mRNA (B) and PRKD protein (C) were detected in each group. (D) Immunofluorescence staining was used to detect the level of PRKD2 from each group. (E) m6A modification sites of PRKD2 was predicted by SRAMP. (F) m6A RNA methylation of C2C12 cells after transfection with METTL3 siRNA was assessed using the m6A RNA Methylation Quantification kit. (G) C2C12 cells were transfected with METTL3 siRNA for $24 \mathrm{~h}$ before treatment with $250 \mu \mathrm{M}$ PA, and PRKD2 protein expression in each group was analyzed with Western blotting. $(\mathrm{H})$ The fluorescence intensity Of PRKD2 in each group. $(\mathrm{N}=3)$ Data are expressed as the mean \pm SEM. $* * ~ P<0.05$ compared with control group, PA group, $\mathrm{PA}+10 \mu \mathrm{M}$ QUE+vector group or PA + Scramble group. 


\section{Figure 7}

Silence of PRKD2 reversed the improvement effect of QUE on PA-induced glucose uptake and oxidative stress through GLUT4/IRS-1/AKT pathway. C2C12 cells were transfected with PRKD2 siRNA before treatment with $250 \mu \mathrm{M}$ PA or $250 \mu \mathrm{M}$ PA with $10 \mu \mathrm{M}$ QUE. (A-B) The protein level of PRKD2 in C2C12 cells were detected using Western blot assay. (C) 2-NBDG uptake of C2C12 cells. The levels of SOD (D), GSH $(E)$, and ROS $(F)$ were detected in each group. $(G-H)$ Western blotting was used to detect the protein levels of GLUT4, IRS1, p-IRS1, AKT and p-AKT. ( $N=3)$ Data are expressed as the mean \pm SEM. ** $P<0.05$ compared with control group, PA group, PA group +insulin group, PA+10 $\mu \mathrm{M}$ QUE group, PA+10 $\mu \mathrm{M}$ QUE +vector group or PA+10 $\mu \mathrm{M}$ QUE+ Scramble group.

\section{Figure 8}

Overexpression of METTL3 or silence of PRKD2 reversed the improving effects of QUE on HFD-induced metabolic disorders and IR. Fasting blood glucose (A), plasma TG (B), serum insulin (C) and HOMA-IR (D) in each group ( $n=8$ per group). Blood glucose levels (E) and AUC (F) of HFD mice in response to the treatment of QUE according to GTT. Blood glucose levels $(\mathrm{G})$ and AUC $(\mathrm{H})$ of HFD mice in response to the treatment of QUE according to IRT. (I-J) Western blotting was used to detect the protein levels of METTL3, PRKD2, GLUT4, IRS1, p-IRS1, AKT and p-AKT.

The levels of SOD $(K), G S H(L)$, and ROS (M) in each group were detected. (N=3) Data are expressed as the mean \pm SEM. ${ }^{*} P<0.05$ compared with HFD+QUE+Lenti-NC+Scramble group.

Figure 9

Schematic representation illustrating the effects of QUE on the progression of IR through METTL3mediated N6-methyladenosine modification of PRKD2 mRNA and oxidative stress.

\section{Supplementary Files}

This is a list of supplementary files associated with this preprint. Click to download.

- supplementarymaterials.docx 